\title{
EL UROCITOGRAMA EN OBSTETRICIA
}

Dr. Alberto Duarte Contreras, M.D.*

Dr. Ramiro Zúñiga Gamboa, M.D.**

Dr. Manuel José Palau C., M.D.***

VI CONGRESO COLOMBIANO DE OBSTETRICIA Y GINECOLOGIA - Cartagena 1965

Desde el año de 1962 hemos tenido la inquietud de utilizar el urocitograma como índice para valorar la normal o anormal evolución del embarazo.

Basados en los estudios y experiencias de Lencioni (1), (2), (3), Cardona Arango (4), López Escobar (5), Alvarez Bravo (6) y otros (7), (8), iniciamos nuestro trabajo, convencidos de la importancia del Urocitograma como método de investigación de la función endocrina placentaria, considerada por nosotros como la más importante investigación que debemos practicar en toda mujer embarazada.

\section{MATERIAL $Y$ METODOS}

Hemos estudiado 306 pacientes del Servicio de Maternidad del Hospital San Juan de Dios de Cúcuta, emba-

* Médico Jefe del Departamento de Obstetricia y Ginecología del Hospital San Juan de Dios de Cúcuta.

** Médico Adjunto del Departamento de Obstetricia y Ginecología del Hospital San Juan de Dios de Cúcuta.

*** Médico Residente del Departamento de Obstetricia y Ginecología del Hospital San Juan de Dios de Cúcuta. razadas y puérperas: ciento cuarenta y cuatro con evolución normal mediante 526 urocitogramas y ciento sesenta y dos de evolución patológica mediante 922 extendidos del sedimento urinario.

Estas experiencias las hemos realizado con la aspiración de poder obtener signos de alarma en los embarazos aparentemente normales, o una ayuda más, fuera de la clínica y los exámenes complementarios en el curso de gestaciones patológicas.

Hemos utilizado preferencialmente la primera orina de la mañana, tomada sin sonda; pero en los casos patológicos se tomaron muestras de orina en el curso del día o de la noche, en casos de grandes hemorragias utilizamos la sonda.

La coloración la hicimos por el método de Shorr (9). En la lectura tuvimos en cuenta los porcentajes de células eosinófilas superficiales 0 profundas, cianófilas intermedias o profundas, porcentaje de picnosis, células intermedias, parabasales, basales y naviculares, presencia de eritrocitos, leucocitos y moco, intensidad de agrupación, plegamiento y citolisis. 


\section{EXPERIENCIAS CLINICAS}

\section{Embarazo normal}

De manera similar a la colpocitología, en el urocitograma se encuentra una disminución de los índices eosinofílico y picnótico a medida que el embarazo progresa.

Estudiamos 16 pacientes con 64 urocitogramas en embarazos de seis a diez semanas. La eosinofilia fluctuó cntre el 1\% y el 8 por ciento encontrándose un promedio de 3,3\% para los embarazos de esta edad.

Las células basófilas fluctuaron entre 92 y 99 por ciento con un promedio del $96,7 \%$.

La picnosis fluctuó entre 2 y 10 por ciento, con un promedio de $5,3 \%$.

Las células parabasales disminuyeron notablemente con el embarazo normal y nosotros encontramos un promedio de $0,8 \%$.

En nuestras pacientes normales no encontramos células cianófilas basales y creemos que el promedio de $0,3 \%$ a que se refiere Lencioni (1) al hablar de células profundas haga referencia a las células parabasales.

Desde el punto de vista cualitativo (indicado por cruces en la práctica corriente) la agrupación celular y el plegamiento aumentan en el embarazo normal.

La presencia de citólisis o destrucción celular, leucocitos, eritrocitos y moco, no la consideramos normal y su hallazgo estará condicionado a cualquier patología del tracto urogenital que altera el urocitograma $y$ nos pone sobre aviso a una posible complicación de la gestación.

Todas las fotos se han tomado con contraste de fase para ver mejor la morfología del protoplasma.

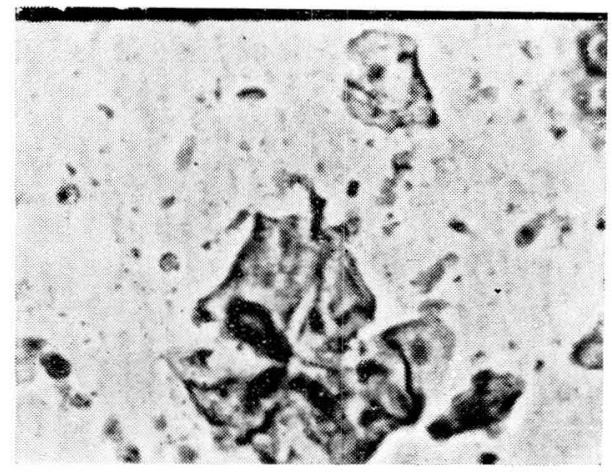

FIGURA 1 - M. 387

Frotis que corresponde a embarazo de ocho semanas.

Embarazo de 11 a 20 semanas. Al estudiar 64 urocitogramas de $16 \mathrm{pa}$ cientes con una gestación normal de 11 a 20 semanas, hemos encontrado un promedio de $3 \%$ de eosinofilia, y un $7 \%$ de picnosis. Las células parabasales disminuyeron a $0,4 \%$. Las células naviculares se encontraron en la casi totalidad de los extendidos; fué intenso el plegamiento y la agrupación, y en algunos casos se encontraron leucocitos y moco seguramente por una colpitis concomitante.

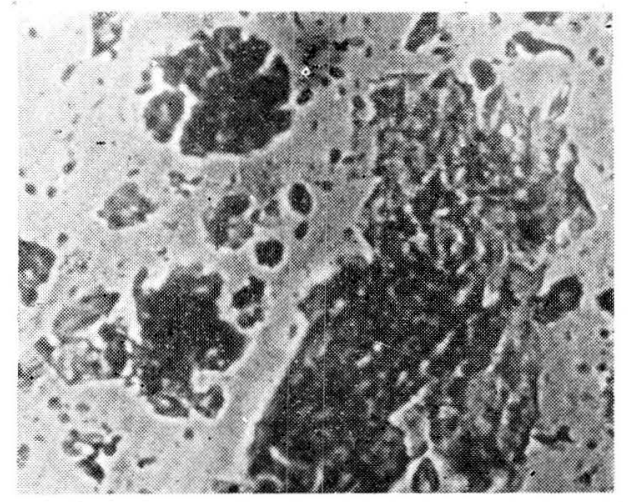

FIGURA 2 - M. 257

Frotis que corresponde a embarazo de veinte semanas. 
Embarazo de 21 a 30 semanas. En el embarazo de 21 a 30 semanas estudiamos 32 pacientes con 124 urocitogramas. Los porcentajes de eosinofilia y de picnosis fueron de 2 y $4 \%$ respectivamente. Se hicieron más abundantes las células naviculares. La agrupación y el plegamiento aumentaron, y hubo en raros casos células parabasales.

Embarazo de $\mathbf{3 0}$ a 35 semanas. Leímos 64 urocitogramas de 16 pacientes con gestación normal de 31 a 35 semanas. Los porcentajes de eosinofilia y de picnosis son sensiblemente iguales a los anteriores, notándose en cambio un discreto aumento en las células parabasales que llegaron al $1 \%$, lo que fué para nosotros una sorpresa ya que no esperábamos encontrarlas en esta proporción. En raros casos encontramos células basales. La agrupación, el plegamiento y las células naviculares aumentaron considerablemente.

En esta edad del embarazo las características morfológicas del frotis siguen siendo las mismas que encontramos en la vigésima semana y solamente se podrían diferenciar en frotis seriados por un aumento progresivo del plegamiento, la agrupación y la presencia de células naviculares a medida que progresa el embarazo.

Embarazo a término. En el embarazo a término estudiamos 22 pacientes con un total de 88 urocitogramas. Encontramos un porcentaje de eosinofilia del $5 \%$ en contra de los hallazgos de Lencioni y colaboradores (1) que dan cifras promedio de $0,69 \%$.

La picnosis aumentó también considerablemente a un promedio de $7 \%$. Las células naviculares eran sin embargo muy abundantes, encontrándose también intensa agrupación y plegamiento. En la mayoría de los extendidos se encontraron abundantes leucocitos y moco. Las células parabasales, cuyo promedio en los meses anteriores había sido mínimo, aumentaron notablemente encontrándose en la proporción de un 3\%. Las células basales que estaban prácticamente ausentes en los urocitogramas de las semanas anteriores, se encontraron en un promedio de $1 \%$.

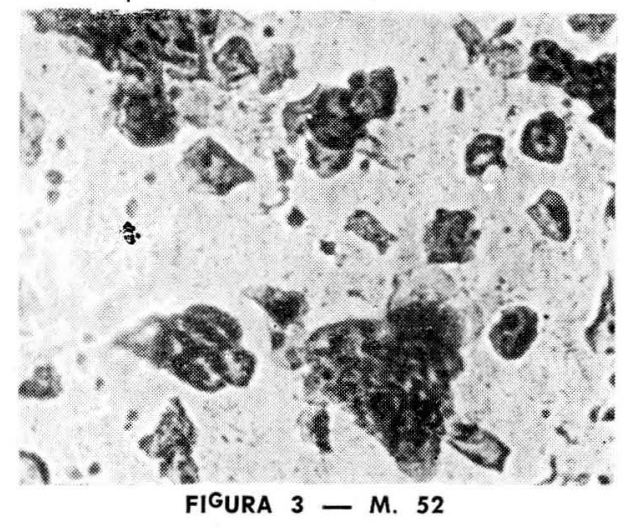

Frotis que corresponde a un embarazo a término.

Trabajo de parto. Estudiamos 37 pacientes en trabajo de parto mediante 148 urocitogramas que nos mostraron un promedio de eosinofilia y de picnosis muy semejantes a los hallados en el embarazo a término. Pero en cambio encontramos un notable aumento de células parabasales y de células basales que llegó a un 5 y un $2 \%$ respectivamente. Disminuyó la agrupación, y las células naviculares que antes eran muy abundantes se hicieron ya muy escasas. El plegamiento permaneció invariable y además del moco y leucocitos encontrados anteriormente comenzamos a notar desintegración celular moderada.

Puerperio. Analizamos 20 pacientes en puerperio con 68 urocitogramas. 
Los porcentajes de eosinofilia y de picnosis iban decreciendo progresivamente, notándose además una disminución apreciable de las células intermedias tanto más acentuada cuanto mayor era el tiempo de postparto. Aumentaban las células parabasales y las células basales, las células naviculares desaparecían y había separación celular encontrándose solo grupos aislados de células con plegamiento disminuído. Hubo abunclante citólisis y leucocitos y en algunos casos eritrocitos y moco.

En el postparto tardío las células que se encontraban eran intermedias - profundas, desapareciendo completamente la picnosis y la eosinofilia. Las células adquirían un contorno más regular con tendencia a ser redondeadas, de núcleos más grandes en relación al citoplasma, y aparecían las células basales cianófilas características. En la mayoría de los casos había leucocitos y citolisis. La agrupación celular y el plegamiento desaparecian y el frotis iba tomando las características de un extendido hipotrófico o atrófico a medida que el puerperio avanzaba para dar lugar al frotis típico de la lactancia.

\section{Embarazo patológico}

Estamos convencidos de que en la vigilancia del embarazo patológico el urocitograma adquiere su mayor importancia ya que los frotis seriados nos pueden mostrar una función hormonal normal o una curva de alarma también llamada amenaza citológica de interrupción del embarazo. Es guía muy importante en la valoración de la eficacia del tratamiento, en la clarificación del diagnóstico sobre posible muerte fetal y en los casos en que el feto es viable es un complemento de la clínica que nos puede indicar la necesidad de interrumpir el embarazo. Así obtendre- mos un niño sin los agravantes consecutivos al sufrimiento endouterino con mayores y mejores posibilidades de supervivencia.

Amenaza de aborto. En el campo de la patología obstétrica es sin duda alguna en la amenaza de aborto donde el urocitograma alcanza su máxima aplicación y utilidad. La amenaza citológica de aborto se caracteriza por un aumento en la eosinofilia $y$ en la picnosis, por disminución o separación de las células naviculares, por la desintegración de los grupos celulares, por alteraciones del plegamiento, por el aumento de las células parabasales y basales cianófilas, en algunos casos, según nuestras observaciones y por el aflujo de moco, leucocitos y desintegración celular.

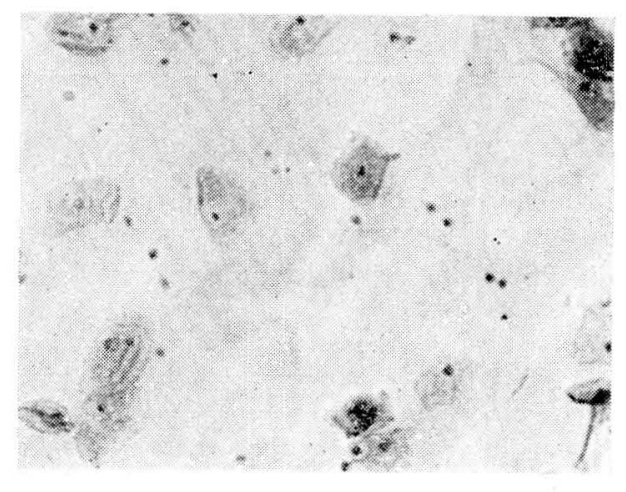

FIGURA $4-$ M. 678

Se aprecia disminución del plegamiento y agrupación celular, con aumento en la picnosis.

Cuanto más elevados sean los porcentajes de eosinofilia y de picnosis - el número de células cianófilas profundas, tanto más sombrío será el pronóstico de la gestación. La práctica diaria nos ha demostrado que no todos los abortos espontáneos obedecen a causas hormonales y es muy frecuente encontrar citologías 
perfectamente normales concomitantes con amenaza de aborto o aborto incompleto reciente. Cuántos de estos abortos serán provocados?, de causa síquica?, de cuello incompetente. o de otras causas?

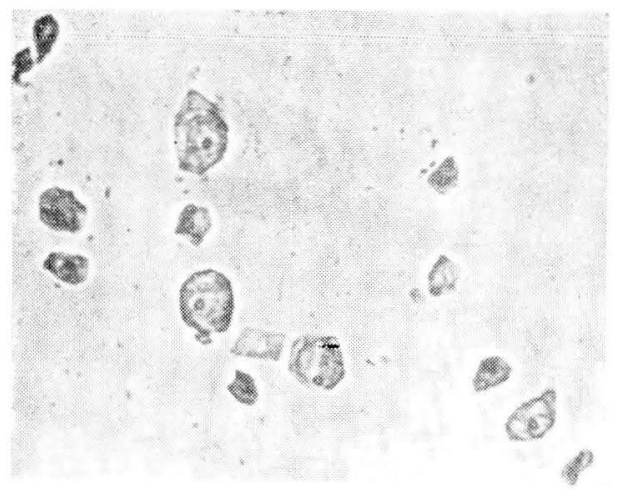

FIGURA $5-$ M. 519

Se aprecia aumento de la cosinofilia y de la picnosis.

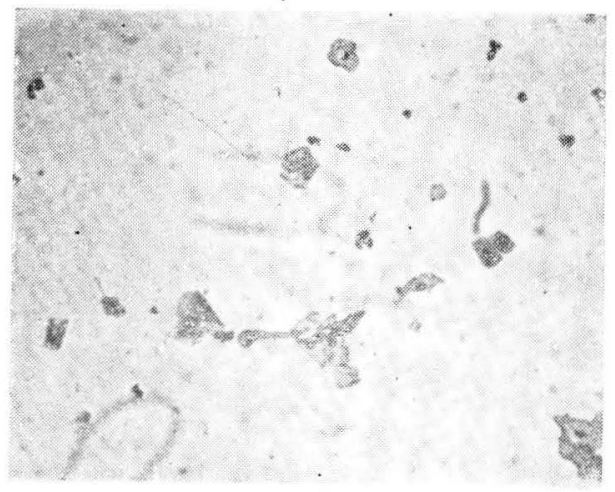

FIGURA 6 - M. 19

Se aprecia un frotis de gestación normal en una paciente con amenaza de aborto. Aborto no hormonal.

Decíamos que el urocitograma o el colpocitograma nos podían dar un indicio en el pronóstico de la gravidez; prueba de ello es el Test de Wateville ya bien estudiado entre nosotros por Cardona Arango y colaboradores (4) cuya interpretación es la siguiente: si al administrar estrógenos el frotis se convierte en más estrogéni$\mathrm{Co}$, el pronóstico es malo o no hay embarazo; si por el contrario la citología se hace progestacional con los estrógenos, el pronóstico es bueno y seguramente la gestación continuará su evolución normal.

Consideramos que la administración precoz de progesterona puede a veces ser innecesaria y en otros casos enmascarar la imagen citológica ocultándosenos la realidad clínica. Por este motivo nosotros no aplicamos progesterona sin el urocitograma previo y en los casos en que hemos podido hacerlo, hemos utilizado el test de Wateville con el fin de orientar correctamente el tratamiento.

Estudiamos 48 pacientes en quienos efectuamos 267 urocitogramas obteniendo datos muy diversos e interesantes. El grupo más numeroso que comprendió 38 casos presentaba un promedio de eosinofilia del $28 \%$ y un promedio de picnosis superior a $35 \%$. En algunos casos a pesar de presentarse una descamación superficial muy numerosa encontramos también células parabasales entre el 5 y el 10\%. Las células naviculares estuvieron ausentes, disminuyeron la agrupación y el plegamiento; encontramos leucocitos, a veces eritrocitos y en raras oportunidades moco. De este grupo abortaron 25 pacientes a pesar del tratamiento.

El grupo restante de 10 pacientes mostró una descamación celular superficial dentro de los límites observados en los embarazos normales pero obtuvimos un aumento notable en la descamación profunda: $15 \%$ de células parabasales y $4 \%$ de células basales.

La evolución clínica fué igualmente desfavorable; abortaron seis. 
Con esta observación corroboramos investro concepto expuesto anteriormente de que el aumento de células basales y parabasales es síntoma de alarma citológica.

Aborto incompleto. Estudiamos 276 urocitogramas de 46 pacientes con aborto incompleto. En el $50 \%$ de los casos encontramos los mismos porcentajes y características que obtuvimos en la amenaza de aborto. En el $21 \%$ encontramos células parabasales y basales, con eosinofilia y picnosis normal, lo que interpretamos como alarma citológica. En el $29 \%$ restantes no encontramos alteración alguna del urocitograma, lo que interpretamos como posibles abortos provocados, lo que pudimos comprobar en cuatro casos por la aseveración de las pacientes. Lógicamente aquí estarían otros casos de aborto de origen no hormonal.

Toxemias. Estudiamos 25 casos de toxemia con 150 urocitogramas; de ellas ocho presentaban toxemia grave.

En el $20 \%$ de los casos de toxemia leve obtuvimos aumento de la cosinofilia, de la picnosis y también de las células cianófilas profundas. En el $80 \%$ restantes hubo evidencia de células parabasales en muy discreta cantidad sin mayor alteración del extendido.

En la toxemia grave encontramos que el promedio de eosinofilia alcanzó en algunos casos a un $10 \%$ con un $14 \%$ de picnosis, un $8 \%$ de células parabasales y un $3 \%$ de células basales. Hubo pérdida de las células naviculares, disminución de agrupación y plegamiento, intensa citolisis, moco y leucocitos.

Cuando la toxemia es grave y hay viabilidad fetal, el urocitograma puede ser de inmenso valor para llevar- nos a interrumpir la gestación mejorando enormemente el pronóstico materno y fetal; pero desafortunadamente estos cambios acentuados no siempre se encuentran.

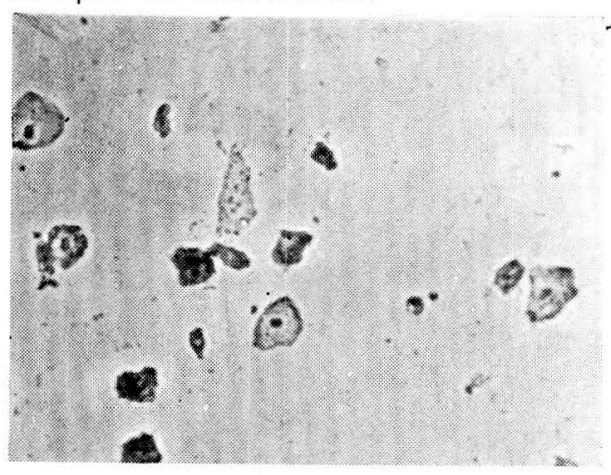

FIGURA 7 - M. 349

Frotis menos progestacional que el normal.

Embarazo ectópico. Doce pacientes con embarazo ectópico roto demostraron en 41 urocitogramas seriados porcentajes de eosinofilia y de picnosis relativamente normales, pero en cambio en todos ellos hubo un discreto aumento de las células parabasales y basales con disminución de las características citológicas de la gestación normal.

Pero el hecho de encontrar estos cambios en nada ayuda al diagnóstico citológico del embarazo ectópico.

Mola hidatiforme. Estudiamos cuatro casos de mola con 24 urocitogramas, encontrando en tres de ellos un aumento en las células cianófilas profundas. El caso restante era normal desde el punto de vista citológico.

En el estudio de nuestras pacientes estamos en desacuerdo con Lencioni quien relata aumento de la eosinofilia y la picnosis en la mayoría de sus casos. 
Muerte fetal. Se han descrito en los frotis de muerte fetal aumento de la eosinofilia y la picnosis, lo mismo que la presencia de células parabasales y basales que cuando son eosinófilas proporcionan el mejor índice de muerte fetal.

Nosotros, en 78 urocitogramas tomados en 13 pacientes con feto muerto, encontramos una gran pérdida de los caracteres progestacionales del frotis. En algunos casos nuestros extendidos se acercan mucho a la descripción clásica encontrando la eosinofilia de las células basales y alteraciones en el protoplasma como son las vacuolas que llegan a ser de mayor tamaño que el núcleo.

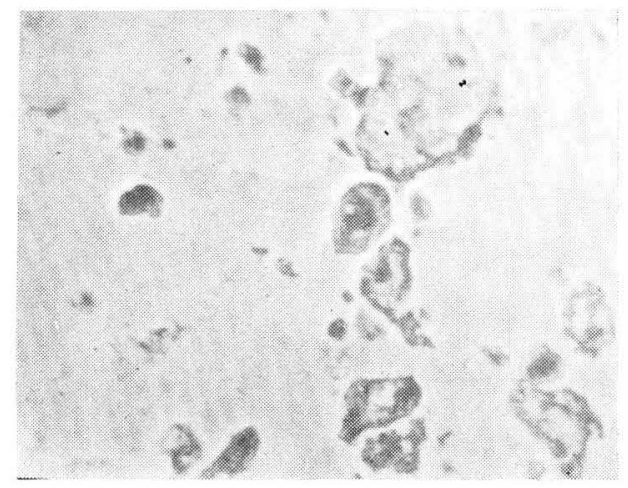

FIGURA $8-$ M. 283

Se aprecia disminución marcada de los caracteres progestacionales del frotis.

\section{CONCLUSIONES}

Estamos convencidos de que el estudio de la función hormonal de la gestante es sin duda alguna el más importante que se deba llevar a cabo durante el embarazo con miras no solo a la supervivencia fetal tanto inmediata como tardía sino también al pronóstico materno; y de que el urocitograma es el método más accesible para efectuar este estudio.

\section{RESUMEN}

Hemos presentado un estudio somero sobre el urocitograma en obstetricia en 306 pacientes con 1.448 extendidos, convencidos de su gran importancia en el manejo de la gestante normal o patológica.

Nuestro único fin ha sido demostrar que en cualquier medio se puede utilizar el urocitograma.

\section{BIBLIOGRAFIA}

1 LENCIONI LEO J.; MARTINEZ AMEZAGA L. A.; LOBIANCO VICTOR S.; OBERTI DE VIALE MARIA R. Y ALONSO CLAUDIO: EI Urocitograma en el embarazo normal y patológico. Rev. Col. Obst. Gin., 6:451, 1964

2 LENCIONI LEO J.: EI Urocitograma. Ed. Médica Panamericana. Buenos Aires 1963.

3 LENCIONI LEO J. El Colpocitograma. Ed. Médica Panamericana. Buenos Aires. 1964.

4 CARDONA ARANGO FERNANDO; RESTREPO JORGE A. Y VELEZ ANTONIO. Citología Vaginal. Su importancia en el embarazo normal, en la amenaza de aborto y en la muerte fetal. Rev. Col. Obst. Gin. 2:288, 1960.

5 LOPEZ ESCOBAR G. Y MENDOZA CESAR. Urocitograma y parto (futuro en Obstetricia). Rev. Col. Obst. Gin. 5:501 1958.

6 ALVAREZ BRAVO A.; GONZALEZ RAMOS M.; GUTIERREZ MURILLO E. Y DOSAL DE LA VEGA. La citología vaginal y la citología del sedimento urinario en el embarazo. Rev. Ginec. Obst. México. 12:73, 1957.

7 Actas del Simposio sobre citología exfoliativa en el embarazo normal y patológico. Rosario, Argentina. 1962

8 BERNASCONI ERNESTO H.; BUS GRATO E. y colaboradores. Simposio de citología exfoliativa en el embarazo normal y patológico. Ed. Médico Panamericana. Buenos Aires 1964.

9 PUNDEL J. PAUL. VAN MEENSEL FRED $y$ JAWORSKI Z. Gestation et cytologie vagina. les. Ed. Masson y Cía. París. 1951. 